then inadequate to force sufficient blood into the muscular fibre to give it tone to act. This defective power of will the stimulus of acupuncture supplies, by producing a temporary congestion and corresponding increase in the calibre of the ressels and in the blood-supply. Two facts seem to point to this explanation: one, that on puncturing the skin we often find, in the course of a minute or so, an area of redness-i. e., of vascular excitement-of about an inch or more in radius; the other, that the recovery of muscular power is gradual, not sudden, beginning immediately after the puncture, and continuing and increasing for twenty or thirty minutes. Moreover a want of power depending upon the wasting of the muscles could not be recovered from in such a short space of time.

2. In relief of pain.-The following seems to me a likely explanation of the manner in which pain is relieved by acupuncture. The injury or inflammation of the offending fibrous tissue has produced a defective nutrition of the nerves pervading such tissue. These nerves are starved of their proper supply of blood, the vasa nervorum being morbidly contracted from pressure or other cause, and the nerves are ill-nourished and in consequence painful. The needle produces a temporary active congestion-a flushing of the vasa nervorum, and thus acts as a starting-point for improved nerve nutrition and for consequent cessation of pain. Such an explanation of relief of pain by acupuncture seems to me in harmony with the views of the causation of neuralgia discussed by Professor Rolleston in the opening of his address on Physiology at Oxford.

In conclusion, let me refer those interested in the subject of this paper to the article "Acupuncture" in the Cyclopædia of Surgery by the late Dr. Elliotson. I may also add the following from a friend many years my senior, who writes to me: "I remember at the Birmingham Hospital it was all the fashion at one time. We used to stick halfa-dozen needles into the deltoid, e.g., and leave them for a quarter of an hour, with sometimes 'wonderful' results."

Leeds, March, 1871.

ON

\section{THE CONDITION OF THE CIRCULATION IN SCROFULA.}

BX WM. KNIGHT TREVES, F.R.C.S.,

visitiNG SURGION, AND LATR RESIDENT SURGEON, TO THE NATIONAL HOSPITAL FOR SCROFUIA.

WHILST making these few observations on the condition of the circulation in scrofula, I must, to avoid misunderstanding, briefly explain what I mean by scrofula, and how it is to be distinguished from those pathological conditions which are usually confounded with it. The first and most important of these is tuberculosis; and I must endeavour to draw a distinction between tuberculosis and scrofula, as the state of the circulation which is found in scrofula does not exist in tuberculosis.

Before describing the chief pathological condition existing in scrofula, I would express my firm belief (for which I will in a future communication bring forward my evidence) that, after putting on one side tuberculosis and hereditary syphilis as totally distinct diseases, there is no evidence whatever of any specific taint in scrofula; that, on the contrary, there is every reason to believe that scrofula is merely slow inflammatory action of an unusually chronic character, produced by slight causes or arising spontaneously in persons predisposed to take on this action by constitutional debility, as evidenced principally by certain defects in the performance of the function of circulation.

The distinguishing pathological condition in a scrofulous person is a peculiar deficiency or weakness in the circulation. The blood appears, from some cause or other, to stagnate, especially in the exposed and superficial portions of the body, causing an appearance of venous congestion. This congestion, indicated on the cheeks and extremities by a purple or bluish tinge mingled with the natural colour, or by red of various shades disposed somewhat patchily, is one of the best signs of scrofula, and is, in fact, an unfailing indicator either of the disease itself or of a predisposition to its attacks. Occasionally a mottled appearance prevails over the whole body. The complexion thus caused varies from the over-fiorid hue of the apparently hearty child, in whom perhaps no consequences have arisen from his constitutional condition, or merely some ailment which does not affect his health, to the dusky tinge mingled with white of the exhansted sufferer. This colour in the cheek, which may sometimes be described as peach-coloured, is distinguished from that of health by the latter's difference of hue, more gradual shading off, greater uniformity of tinge, and clearness of surrounding skin. The scrofulous person is generally rather dusky, and even in cases where this duskiness is not shown in the face, and where the com. plexion may be bright if overloaded with colour, the constitutional condition may be revealed by the hands, the backs of which almost invariably exhibit the blue and mottled appearance I have described. A further distinction is that, if the scrofulous person has a bright colour, it appears to be on the surface, is abrupt, and obtrudes itself on one's notice, while the same amount of colour in a healthy person is more diffused and not so remarkable.

Another peculiarity in scrofulous individuals, due also to the condition of their circulation, is a swollen appearance of their extremities; they generally look as if they were suffering from extreme cold, and this appearance of the hands and feet remains, to a greater or less extent, even in very hot weather. Their legs and feet of ten have a doughy feel, and lack the firm resiliency of health.

Now this venous congestion exists not only in the skin and external surface of the body, but also in the mucous membranes, and is the predisposing cause, in a typical case of scrofula, not only of the disease itself, but even of the very appearance of the patient. The pulse is below the average of health, of fair volume, soft and regular, but wanting in force. The temperature is certainly not raised, and I am inclined to believe, from some observations I am making, that it is below that of health.

Scrofulous people are, as a rule, fleshy and heavy-looking, although not wanting in intellect; they are flabby and often deficient in muscular power. Their lips, alæ of nose, and eyelids are generally tumid from congested mucous mem. brane, and their abdomen is inclined to be prominent. Their extremities are large and swollen, and do not usually exhibit that symmetry which may be found in healthier persons. Their hair may be any colour; the red and the dark hair show the best specimens of scrofula, but the dark are the more numerous. They do not in any particular resemble people suffering from tubercular disease, nor have they any tendency to become tuberculous. They do not waste, nor do they suffer from that irritable form of dyspepsia known as strumous dyspepsia. Their circulation is not quickened, nor their temperature raised. They have not the quick, irritable pulse of tuberculosis ; nor have they, even in advanced cases, any fever or night sweats. Their disease does not run a rapid course, but generally extends over many years, and very rarely proves fatal. They are good subjects for operation, exhibiting no irritability, the only drawback being that their wounds have a tendency to become sluggish. In these and numerous other symptoms they are diametrically opposite to persons suffering from tubercular disease; and I hope shortly to be able to lay these distinctions, drawn from observations in a large number of cases, more clearly and fully before the profession.

Margate, April, 1871.

\section{FATAL CASE OF FRACTURE OF CERVICAL VERTEBRA.}

By Def. Inspector-General J. L. DONNET, M.D., R.N., ROYAL MAVAL HOSPITAI, PORT ROYAX, JAMAICA.

(Communicated by the Director-Gentral of the Medical Department of the Navx.)

AN officer, on the morning of the 15th of February, had gone to Port Henderson for the purpose of bathing. (Port Henderson is situated on the opposite shore, two miles from Port Royal.) In the act of plunging head foremost, his head struck the sandy bottom with some force, and when assistance was afforded he was discovered to be powerless in his lower limbs. He was brought into Jamaica Hospital at 9.45 on the morning of the accident. 
On examination, there was complete paralysis both of sensation and of motion of the body, commencing at a line which surrounded the thorax about two inches and a half below the nipples. The head was perfectly free; intellect clear; he had remembrance of everything that had occurred and of all the circumstances connected with his state. The upper extremities were under the control of his will, especially the shoulders and arms; but the forearm appeared to have lost some degree of power, for, as he raised the arm, the forearm, though raised with it, soon after flexed itself on the elbow, and the hand was remarked to have lost its power of grasping. The sensation of the upper extremities was intact. On running the hand along each vertebra of the spinal column, he made no complaint of pain until the hand came in contact with that portion which corresponded to the fourth, fifth, sixth, and seventh cervical vertebræ the hand, however, could discover no displacement nor dislocation in this locality. The rotation of the head upon the spine was easy, but any attempt at flexion was accompanied by pain. The pulse was natural, the respiration normal, the expression of the face good; no sensation could be elicited by tickling the soles of the feet; there was marked priapism. He was placed in a water bed, the head adjusted in such a way as to control all movement; the urine was drawn off by catheter, and the bowels relieved by enemata.

On the evening of the 18th he complained more of his back; the pulse was somewhat higher; there was a feeling of cold, which he referred to a fiery sea-breeze which was then blowing, and which was felt through the whole building.

On the morning of the 19 th the temperature had risen to $105^{\circ}$, and the beats of the pulse to 106 ; his mind wandered, and he ejected from his stomach a large quantity of matter resembling black vomit. (This matter, when placed under the microscope, was of a reddish-brown colour, and appeared to consist chiefly of numerous non-nucleated globules intermixed with some epithelium of the stomach.) The romiting continued for some time, but was at first partly, then wholly, checked by means of effervescing draughts, champagne, ice, \&c., and did not recur after 2 P.M. Albuminous urine coexisted with this vomit.

Though the patient had slept during the greater part of the night of the 19th, his rest had been troubled by dreams. There was great prostration; his intellect, however, had become again clear, and continued so to within a short time before death. The pulse was 100 ; temperature of body $104^{\circ}$; respiration laboured and sibilant; pupils contracted. The vital powers were fast diminishing; convulsive twitchings of the muscles of the face were observed, followed by gentle retraction of the forearms, and as gentle relaxation; and at 2.45 P.M. he expired. At the time of the convulsive twitchings of the muscles of the face and of the drawing up of the forearms the pulse beat 72 per minute with much regularity, and the respiratory movements were four per minute.

At the post-mortem examination, which took place sixteen hours after death, the fifth cervical vertebra was found fractured transversely, close to its articulating facets, so that the spinous process with its laminæ was quite detached from the body of this vertebra; the body itself was broken up into a number of small pieces. The spinal cord had suffered much compression opposite to the fifth cervical vertebra, and was, at this point, almost wholly converted into a mass of blood and soft spinal matter. A quantity of blood had been effused into the substance of the cord above and below this portion and had obliterated the grey matter. This effusion had extended upwards to the upper edge of the third cervical vertebra, and downwards to the lower edge of the first dorsal. The greater portion of this part of the spinal cord was disintegrated, and had become softened where it had come in contact with the effused blood. There was no effusion of blood between the cord and theca; the pia mater investing the spinal cord was here hyperæmic. The mucous coats of the stomach and intestines were highly congested, and in the cavity of the former there was a quantity, amounting to six ounces, of a black grumous matter. The liver was of a healthy appearance in colour and consistence, whilst the spleen was larger than natural, and, under pressure, broke down into a matter resembling red currant jelly, though darker. The urine in the bladder was highly albuminous, and contained a quantity of phosphates.
In the symptoms which marked the case two offered which were peculiar-viz., black vomit and albumen in the urine. I can only refer these symptoms to the great shock that the nervous system had sustained, which, acting upon the cerebro-spinal axis in a manner analogous to that of the poison of yellow fever, had produced similar results; for with the exception of these two symptoms none other offered. The liver was natural; and though the heat of surface was above the normal line of temperature, this was accounted for by the hyperæmic state of the coverings of the spinal cord, and by the congested state of the mucous coats of the stomach and intestines.

\section{NOTES OF A CASE OF HEPATIC ABSCESS.}

Br G. W. JAMESON, ASSISTANT-SURGEON, H.M. INDIAN MEDICAI SERVICE.

A MahommedaN FAKEER, aged forty, was admitted into hospital at Hurdui, Oudh, under my care, on the 23rd of August, 1865, complaining of pain in his right side. He said he had been ill for about four months with intermittent fever, and at time of admission was greatly emaciated. There was considerable enlargement of the liver, pain on pressure, rigidity of the muscles of the chest and abdomen on the right side, constipated bowels, and peculiarly dark, offensive stools. No history of dysentery. Ordered, quinine, nitro-hydrochloric acid, one-third of a grain of podophyllin, with ipecacuanha and henbane, every night, and the application of iodine externally.

Aug. 28th.-Says he feels a good deal easier; stools more natural; liver smaller if anything, and less painful on pressure.

Sept. 2nd.-The hepatic tumour has begun to bulge more than before, just below the edge of the ribs; indistinct fluctuation; suspiciously hectic-like symptoms have come on.

4th.-Fluctuation more distinct; hectic more marked. Decided to operate. A broad abdominal bandage having been first firmly applied (a small hole being left in it at the seat of puncture), the tumour was then tapped at its most prominent part, a little below the edge of the ribs, with a medium-sized ordinary trocar and canula, and seventy-two fluid ounces of purulent fluid of a pinkish tinge were drawn off. The canula being then found to be grasped in the wound in the liver, the abscess was gently syringed out with tepid water containing a small quantity of solution of permanganate of potass, and the instrument secured in the wound by means of strips of adhesive plaster, the patient being placed on his right side.

5th.-Feels much easier; pulse weak, but not fast. Abscess syringed out as before, twice daily; the canula remains firmly in the wound. To have stimulants and nourishing diet.

6th.-Easy, but weak; discharge from canula has been gradually decreasing; is quite free from smell. Abscess washed out as before.

7th.-Canula dropped out of wound; no discharge; no pain; very weak.

8th.-Morning: rather weaker, but comfortable. Stimulants freely given. Puncture healed up.-Evening: died of exhaustion.

An autopsy was made a few hours after death; and it was discovered that a single abscess occupied the whole substance of the liver, except a small portion of the posterior part of the right lobe. The liver presented the appearance of a bag, with walls in some places not more than a quarter of an inch thick. The cavity of the abscess contained a small quantity of pus, similar to that which had been removed during life. This cavity was found capable of containing eighteen fluid ounces of water. The walls of the abscess were studded with numerous granulations. There were adhesions to the stomach and diaphragm, and to the abdominal wall where the abscess had been punctured. The wound had been obliterated by recent plastic effusion; and the adhesions only extended to about half an inch round the course of the trocar. The gall-bladder was quite empty, but apparently healthy. The body was greatly 